\title{
A code based hybrid signcryption scheme
}

This paper was downloaded from TechRxiv (https://www.techrxiv.org).

\section{LICENSE}

CC BY 4.0

SUBMISSION DATE / POSTED DATE

$19-12-2021 / 28-12-2021$

CITATION

KLAMTI, Jean Belo; Hasan, Anwar (2021): A code based hybrid signcryption scheme. TechRxiv. Preprint. https://doi.org/10.36227/techrxiv.17283899.v1

DOI

10.36227/techrxiv.17283899.v1 


\title{
A code-based hybrid signcryption scheme
}

\author{
Jean Belo KLAMTI and M. Anwar Hasan
}

\begin{abstract}
A key encapsulation mechanism (KEM) that takes as input an arbitrary string, i.e., a tag, is known as tag-KEM, while a scheme that combines signature and encryption is called signcryption. In this paper, we present a codebased signcryption tag-KEM scheme. We utilize a code-based signature and a CCA2 (adaptive chosen ciphertext attack) secure version of McEliece's encryption scheme. The proposed scheme uses an equivalent subcode as a public code for the receiver, making the NP-completeness of the equivalent subcode problem be one of our main security assumptions. We then base the signcryption tag-KEM to design a code-based hybrid signcryption scheme. A hybrid scheme deploys an asymmetric- as well as a symmetric-key encryption. We give security analyses of both our schemes in the standard model and prove that they are secure against IND-CCA2 (indistinguishability under adaptive chosen ciphertext attack) and SUF-CMA (strong existential unforgeability under chosen message attack).
\end{abstract}

\section{Index Terms}

Coding theory, signature scheme, public-key cryptography, code-based cryptography, signcryption.

\section{INTRODUCTION}

In public-key cryptography, the authentication and confidentiality of communication between a sender and a receiver are ensured by a two-step approach called signature-then-encryption. In this approach, the sender uses a digital signature scheme to sign a message and then encrypt it using an encryption algorithm. The cost of delivering a message in a secure and authenticated way using the signature-then-encryption approach is essentially the sum of the cost of digital signature and that of encryption.

In 1997, Y. Zheng introduced a new cryptographic primitive called signcryption to provide both authentication and confidentiality in a single logical step [62]. In general, one can expect the cost of signcryption to be noticeably less than that of signature-then-encryption. Zheng's sincryption scheme is based on the hardness of discrete logarithm problem. Since Zheng's work, a number of signcryption schemes based on different hard assumptions have been introduced, see for example [62], [63], [56], [58], [37], [7], [8], [28], [53], [59], [36], [61]. Of these, the most efficient ones have followed Zheng's approach, i.e., used symmetric-key encryption as a black-box component [7], [8], [28]. It has been of interest to many researchers to study how a combination of asymmetric- and symmetric-key encryption schemes could be used to build efficient signcryption schemes in a more general setting.

To this end, Dent in 2004 proposed the first formal composition model for hybrid signcryption [25] and in 2005 developed an efficient model for signcryption KEMs in the outsider-and the insider-secure setting [26], [27]. In the outsider-secure setting the adversary is assumed to be distinct from the sender and receiver, while in the insider-secure setting the adversary is assumed to be a second party (i.e., either sender or receiver). In order to improve the model for the insider-secure setting in hybrid signcryption, Bjørstad and Dent in 2006 proposed a model based on encryption tag-KEM rather than regular encryption KEM [14]. Their model provides a simpler description of signcryption with a better generic security reduction for the signcryption tag-KEM construction. A year after Bjørstad and Dent's work, Yoshida and Fujiwara reported the first study of multi-user setting security of signcryption tag-KEMs [60] which is a more suitable setting for the analysis of insider-secure schemes.

The authors are with the Department of Electrical and Computer Engineering, University of Waterloo, Waterloo, ON, Canada. Email: jbklamti@uwaterloo.ca and ahasan@uwaterloo.ca 
Motivation: Most of the aforementioned signcryption schemes are based on the hardness of either the discrete logarithm or the integer factorization problem and would be broken with the arrival of sufficiently large quantum computers. Therefore it is of interest to design signcryption schemes for the post-quantum era. Coding theory has some hard problems that are considered quantum-safe and in this paper we explore the design of code-based signcryption.

The first attempt for code-based signcryption was presented in 2012 by Preetha et al. [40]. After that work, an attribute-based signcryption scheme based on linear codes was introduced in 2017 by Song et al. [54]. Code-based signcryption remains an active area of research, specifically to study the design of cryptographic primitives like signcryption schemes that are quantum-safe.

Contributions: In this paper we present a signcryption tag-KEM scheme using a probabilistic full domain hash $(\mathrm{FDH})$ like code-based signature and a CCA2 secure version of McEliece's encryption scheme. The underlying code-based signature in our scheme is called Wave introduced by Debris-Alazard et al. [4], while the CCA2 secure version of the McEliece scheme is based on the Fujisaki-Okamoto conversion introduced by Cayrel et al. [16]. Instead of using only the hardness of the Goppa syndrome decoding problem, we add a second security assumption which is the NP-completeness of the subcode equivalence problem [10]. To this end, we use an equivalent Goppa subcode as the receiver public code. Then, we base our signcryption tag-KEM to design a code-based hybrid signcryption scheme. We also give security analyses of these two schemes in the standard model assuming the insider-secure setting.

Organization: This paper is organized as follows. In Section [I] we first recall some basic notions of coding theory and then briefly describe relevant encryption and signature schemes that are of interest to this work. Section III has the definition and framework of signcryption and hybrid signcryption, and a brief review of the relevant security model. We present our sigcryption and hybrid sigcryption schemes in Section IV and then provide security analyses of the proposed schemes in Section $\mathrm{V}$. We provide a set of parameters for the hybrid sigcryption scheme in Section VI and then conclude in Section VII.

Notations: In this paper we use the following notations:

- $\mathbb{F}_{q}$ : finite field of size $q$ where $q=p^{m}$ is a prime power.

- $\mathcal{C}: \mathbb{F}_{q}$-linear code of length $n$.

- $x$ : a word or vector of $\mathbb{F}_{q}^{n}$.

- $w t(\boldsymbol{x})$ : weight of $\boldsymbol{x}$.

- $\mathrm{G}$ (resp. $\mathbf{H})$ : generator (resp. parity-check) matrix of linear code $\mathcal{C}$.

- $\mathcal{W}_{q, n, t}$ is the set of $q$-ary vectors of length $n$ and weight $t$.

- $S C S K_{s}$ (resp. $S C S K_{r}$ ): sender's (resp. receiver's) secrete key for signcryption.

- $S C P K_{s}$ (resp. $S C P K_{r}$ ): sender's (resp. receiver's) public key for signcryption.

\section{PRELIMINARIES}

In this section we recall some notions pertaining to coding theory and code-based cryptography.

\section{A. Coding theory and some relevant hard problems}

Let us consider the finite field $\mathbb{F}_{q}$. A $q$-ary linear code $\mathcal{C}$ of length $n$ and dimension $k$ over $\mathbb{F}_{q}$ is a vector subspace of dimension $k$ of $\mathbb{F}_{q}^{n}$. It can be specified by a full rank matrix $\mathrm{G} \in \mathbb{F}_{q}^{k \times n}$, called generator matrix of $\mathcal{C}$, whose rows span the code. Namely, $\mathcal{C}=\left\{\boldsymbol{x} \mathrm{G}\right.$ s.t. $\left.\boldsymbol{x} \in \mathbb{F}_{q}^{k}\right\}$. A linear code can also be defined by the right kernel of matrix $\mathbf{H} \in \mathbb{F}_{q}^{r \times n}$, called parity-check matrix of $\mathcal{C}$, as follows:

$$
\mathcal{C}=\left\{\boldsymbol{x} \in \mathbb{F}_{q}^{n} \text { s.t. } \quad \mathbf{H} \boldsymbol{x}^{T}=\boldsymbol{0}\right\}
$$

The Hamming distance between two codewords is the number of positions (coordinates) where they differ. The minimal distance of a code is the minimal distance of all codewords. 
The weight of a word or vector $\boldsymbol{x} \in \mathbb{F}_{q}^{n}$, denoted by $w t(\boldsymbol{x})$, is the number of its nonzero positions. Then the minimal weight of a code $\mathcal{C}$ is the minimal weight of all nonzero codewords. In the case of linear code $\mathcal{C}$, its minimal distance is equal to the minimal weight of the code.

Below we recall some hard problems that are relevant to our discussions and analyses presented in this article.

Problem 1: (Binary syndrome decoding (SD) problem) Given a matrix $\mathbf{H} \in \mathbb{F}_{2}^{r \times n}$, a vector $\boldsymbol{s} \in \mathbb{F}_{2}^{r}$, and an integer $\omega>0$, find a vector $\boldsymbol{y} \in \mathbb{F}_{2}^{n}$ such that $w t(\boldsymbol{y})=\omega$ and $\boldsymbol{s}=\boldsymbol{y} \mathbf{H}^{T}$.

The syndrome decoding problem was proven to be NP-complete in 1978 by Berlekamp et al. [13]. It is equivalent to the following problem.

Problem 2: (General decoding (GBD) problem) Given a matrix $\mathbf{G} \in \mathbb{F}_{2}^{k \times n}$, a vector $\boldsymbol{y} \in \mathbb{F}_{2}^{n}$, and an integer $\omega>0$, find two vectors $\boldsymbol{m} \in \mathbb{F}_{q}^{k}$ and $\boldsymbol{e} \in \mathbb{F}_{q}^{n}$ such that $w t(\boldsymbol{e})=\omega$ and $\boldsymbol{y}=\boldsymbol{m} \mathrm{G} \oplus \boldsymbol{e}$.

The following problem is used in the security proof of the underlying signature that we use in this paper. It was first considered by Johansson and Jonsson in [35]. It was analyzed later by Sendrier in [52].

Problem 3: (Decoding One Out of Many (DOOM) problem) Given a matrix $\mathbf{H} \in \mathbb{F}_{q}^{r \times n}$, a set of vector $\boldsymbol{s}_{1}, \boldsymbol{s}_{2}, \ldots, \boldsymbol{s}_{N} \in \mathbb{F}_{q}^{r}$ and an integer $\omega$, find a vector $\boldsymbol{e} \in \mathbb{F}_{q}^{n}$ and an integer $i$ such that $1 \leq i \leq N, w t(\boldsymbol{e})=\omega$ and $\boldsymbol{s}_{i}=\boldsymbol{e} \mathbf{H}^{T}$.

Problem 4: (Goppa code distinguishing problem) Given a matrix $\mathbf{H} \in \mathbb{F}_{2}^{r \times n}$, decide whether $\mathbf{H}$ is a random binary or parity-check matrix of a Goppa code.

Faugère et al. [30] showed that Problem 4 can be solved in special cases of Goppa codes with high rate.

The following is one of the problems, which the security assumption of our scheme's underlying signature mechanism relies on.

Problem 5: (Generalized $(U, U+V)$ code distinguishing problem.) Given a matrix $\mathbf{H} \in \mathbb{F}_{q}^{r \times n}$, decide whether $\mathbf{H}$ is a parity-check matrix of a generalized $(U, U+V)$-code.

Problem 5] was shown to be hard in the worst case by Debris-Alazard et al. [22] since it is NP-complete. Below, we recall the subcode equivalence problem which is the second problem on which the security assumptions of our scheme is based. This problem was proven to be NP-complete in 2017 by Berger et al. [10].

Problem 6: (Subcode Equivalence problem [10]) Given two linear codes $\mathcal{C}$ and $\mathcal{D}$ of length $n$ and respective dimension $k$ and $k^{\prime}, k^{\prime} \leq k$, over the same finite field $\mathbb{F}_{q}$, determine whether there exists a permutation $\sigma$ of the support such that $\sigma(\mathcal{C})$ is a subcode of $\mathcal{D}$.

\section{B. Code-based encryption}

The first code-based encryption was introduced in 1978 by R. McEliece [42]. Below (in Figure 1) we give the McEliece scheme Fujisaki-Okamoto conversion [16] which comprises three algorithms: key generation, encryption, and decryption.

The main drawback of the McEliece encryption scheme is its very large key size. To address this issue, many variants of McEliece's scheme have been proposed, see for example [11], [12], [43], [44], [9], [47]. In order to reduce the size of both public and private keys in code-based cryptography, H. Niederreiter in 1986 introduced a new cryptosystem [45]. Niederreiter's cryptosystem is a dual version of McEliece's cryptosystem with some additional properties such that the ciphertext length is relatively smaller. Indeed, the public key in Niederreiter's cryptosystem is a parity-check matrix instead of a generator matrix. In addition, ciphertexts are syndrome vectors instead of erroneous codewords. However, the McEliece and the Niederreiter schemes are equivalent from the security point of view due to the fact that Problems 1 and 2 are equivalent. 


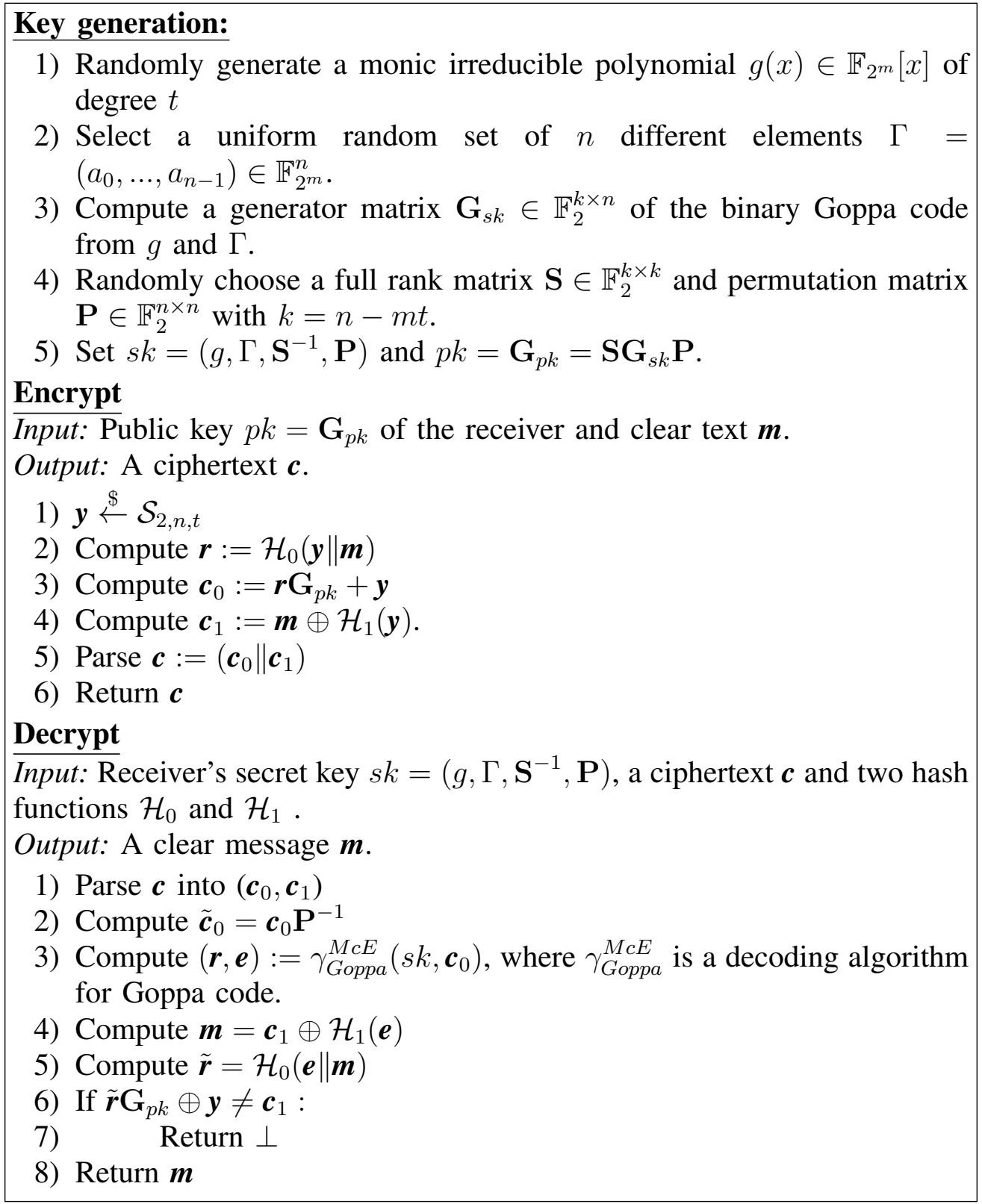

Fig. 1. McEliece's scheme with Fujisaki-Okamoto conversion

Code-based hybrid encryption: A hybrid encryption scheme is a cryptographic protocol that features both an asymmetric- and a symmetric-key encryption scheme. The first component is known as Key Encapsulation Mechanism (KEM), while the second is called Data Encapsulation Mechanism (DEM). The framework was first introduced in 2003 by Cramer and Shoup [21] and later the first code-based hybrid encryption was introduced in 2013 by Persichetti [48] using Niederreiter's encryption scheme. Persichetti's scheme was implemented in 2017 by Cayrel et al. [17]. After Persichetti's work, some other code-based hybrid encryption schemes have been reported, e.g., [41]. 


\section{Code-based signature}

Designing a secure and practical code-based signature scheme is still an open problem. The first secure code-based signature scheme was introduced by Courtois et al. (CFS) [20]. It is a full domain hash (FDH) like signature with two security assumptions: the indistinguishability of random binary linear codes and the hardness of syndrome decoding problem. To address some of the drawbacks of Courtois et al.'s scheme, Dallot proposed a modified version, called mCFS, which is provably secure. Unfortunately, this scheme is not practical due to the difficulties of finding a random decodable syndrome. In addition, the assumption of the indistinguishability of random binary Goppa codes has led to the emergence of attacks as described in [30]. One of the latest code-based signature schemes of this type is called Wave [23]. It is based on generalized $(U, U+V)$-codes. It is secure and more efficient than the CFS signature scheme. In addition, it has a smaller signature size than almost all finalist candidates in the NIST post-quantum cryptography standardization process [5].

Apart from the full domain hash approach, it is possible to design signature schemes by applying the Fiat-Shamir transformation [31] to an identification protocol. To this end, one may use a code-based identification scheme like that of Stern [57], Jain et al. [34], or Cayrel et al. [18]. This approach however leads to a signature scheme with a very large signature size. To address this issue, Lyubashevsky's framework [38] can apparently be adapted. Unfortunately almost all code-based signature schemes in Hamming metric designed by using this framework have been cryptanalyzed [15], [49], [50], [32], [39], [55]. The only one which has remained secure so far is a rank metric-based signature scheme proposed by Aragon et al.[1].

In Figure 2, we recall Debris-Alazard et al.'s signature scheme (Wave) which is of our interest for this work. In Wave, the secret key is a tuple of three matrices $s k=\left(\mathbf{S}, \mathbf{H}_{s k}, \mathbf{P}\right)$, where $\mathbf{S} \in \mathbb{F}_{q}^{r \times r}$ is an invertible matrix, $\mathbf{H}_{s k} \in \mathbb{F}_{q}^{r \times n}$ is a parity-check matrix of a generalized $(U, U+V)$-code and $\mathbf{P} \in \mathbb{F}_{2}^{n \times n}$ is a permutation matrix. The public key is a matrix $p k=\mathbf{H}_{p k}$, where $\mathbf{H}_{p k}=\mathbf{S H}_{s k} \mathbf{P}$. Steps for signature and verification processes are given in Figure 2. For additional details, the reader is referred to [24], [23].

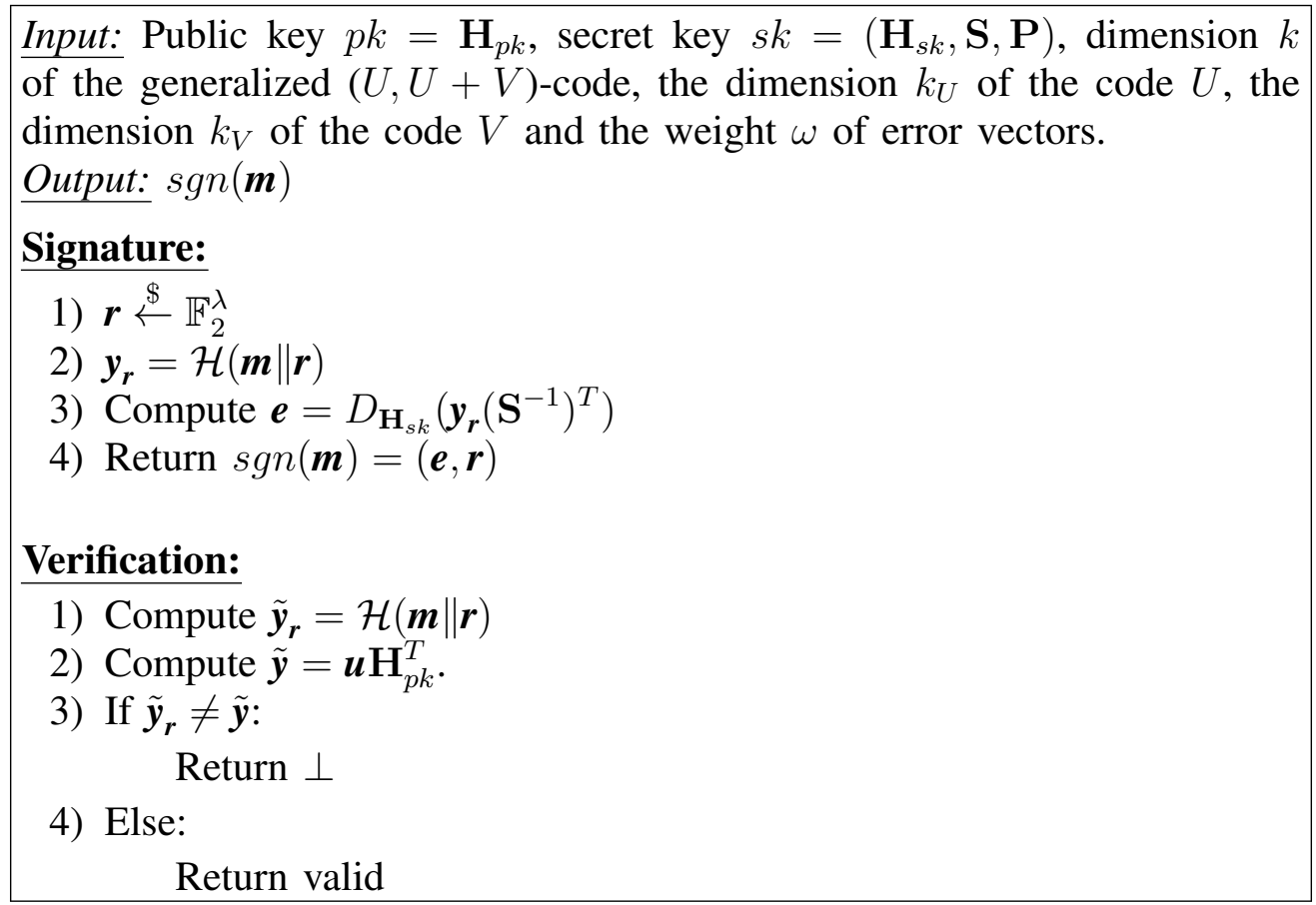

Fig. 2. Wave signature scheme [23] 


\section{SIGNCRYPTION AND SECURITY MODEL}

In this section, we first recall the definition of signcryption followed by the signcryption tag-KEM framework and its security model under the insider setting.

\section{A. Signcryption and its tag-KEM framework}

Signcryption: A signcryption scheme is a tuple of algorithms $S C=\left(\right.$ Setup $_{\text {KeyGen }}, K_{\text {KeyGen }}$, Signcrypt, Unsigncryt) [3] where:

* $\operatorname{Setup}\left(1^{\lambda}\right)$ is the common parameter generation algorithm with $\lambda$, the security parameter,

* KeyGen $\left(\right.$ resp. KeyGen ${ }_{r}$ ) is a key-pair generation algorithm for the sender (resp. receiver),

* Signcrypt is the signcryption algorithm and

* Unsigncrypt corresponds to the unsigncryption algorithm.

For more details on the design of signcryption, the reader is referred to [29] (Chap. 2, Sec. 3, p. 30).

Signcryption tag-KEM: A signcryption tag-KEM denoted by SCTKEM is a tuple of algorithms [14]:

$$
\text { SCTKEM }=\left(\text { Com, KeyGen }, \text { KeyGen }_{r}, \text { Sym, Encap, Decap }\right)
$$

where,

- Com is an algorithm for generating common parameters.

- KeyGen (resp. KeyGen $_{r}$ ) is the sender (resp. receiver) key generation algorithm. It takes as input the global information $I$, and returns a private/public keypair $\left(\mathrm{SCSK}_{s}, \mathrm{SCPK}_{s}\right)\left(\right.$ resp. $\left.\left(\mathrm{SCSK}_{r}, \mathrm{SCPK}_{r}\right)\right)$ that is used to send signcrypted messages.

- Sym is a symmetric key generation algorithm. It takes as input the private key of the sender $\mathrm{SCSK}_{s}$ and the public key of the receiver $\mathrm{SCPK}_{r}$, and outputs a symmetric key $K$ together with internal state information $\varpi$.

- Encap takes as input the state information $\varpi$ together with an arbitrary string $\tau$, which is called a tag, and outputs an encapsulation $E$.

- Decap is the decapsulation/verification algorithm. It takes as input the sender's public key $\mathrm{SCPK}_{s}$, the receiver's private key $\mathrm{SCSK}_{r}$, an encapsulation $E$ and a tag $\tau$. It returns either symmetric key $K$ or the unique error symbol $\perp$.

Hybrid signcryption tag-KEM+DEM: It is simply a combination of a SCTKEM and a regular Data Encapsulation Mechanism (DEM).

\section{B. Insider security for signcryption tag-KEM}

IND-CCA2 game in signcryption tag-KEM: It corresponds to a game between a challenger and a probabilistic polynomial-time adversary $\mathcal{A}_{C C A 2}$ such that the latter tries to distinguish whether a given session key $K$ is the one embedded in an encapsulation or not. During this game, $\mathcal{A}_{C C A 2}$ has an adaptive access to three oracles for the attacked user corresponding to algorithms Sym, Encap, and Decap [14], [29], [60]. The game is described in Figure 3 below.

During Step 7, the adversary $\mathcal{A}_{C C A 2}$ is restricted not to make decapsulation queries on $(E, \tau)$ to the descapsulation oracle. The advantage of the adversary $\mathcal{A}$ is defined by:

$$
\mathcal{A} d v\left(\mathcal{A}_{C C A 2}\right)=\left|\operatorname{Pr}\left(b^{\prime}=b\right)-1 / 2\right| .
$$

A signcryption tag-KEM is IND-CCA2 secure if, for any adversary $\mathcal{A}$, its advantage in the IND-CCA2 game is negligible with respect to the security parameter $\lambda$.

SUF-CMA game for signcryption tag-KEM: This game is a challenge between a challenger and a probabilistic polynomial-time adversary (i.e., a forger) $\mathcal{F}_{C M A}$. In this game, the forger tries to generate 


\section{Oracles:}

1) $\mathcal{O}_{\text {Sym }}$ is the symmetric key generation oracle with input a public key $S C P K$, and computes $(K, \omega)=\operatorname{Sym}\left(\mathrm{SCSK}_{s}, \mathrm{SCPK}\right)$. It then stores the value $\omega$ (hidden from the view of the adversary, and overwriting any previously stored values), and returns the symmetric key $K$.

2) $\mathcal{O}_{\text {Encap }}$ is the key encapsulation oracle. It takes an arbitrary tag $\tau$ as input, and checks whether there exists a stored value $\omega$. If there is not, it returns $\perp$ and terminates. Otherwise it erases the value from storage, and returns $E=\operatorname{Encap}(\omega, \tau)$.

3) $\mathcal{O}_{\text {Decap }}$ corresponds to the decapsulation/verification oracle. It takes an encapsulation $E$, a tag $\tau$, any sender's public key SCPK as input and returns $\operatorname{Decap}\left(S C P K, S C S K_{r}, E, \tau\right)$.

\section{IND-CCCA2 Game for SCTKEM:}

1) $I:=\operatorname{Com}\left(1^{\lambda}\right)$

2) $\left(S C S K_{r}, S C P K_{r}\right):=\operatorname{KeyGen}_{r}(I)$

3) $\left(S C S K_{s}\right.$, state $\left._{1}\right):=\mathcal{A}_{C C A 2}^{\mathcal{O}_{\text {Sym }}, \mathcal{O}_{\text {Encap }}, \mathcal{O}_{\text {Decap }}}\left(S C P K_{r}\right)$

4) $\left(K_{1}, \varpi\right):=\operatorname{Sym}\left(S C S K_{s}, S C P K_{r}\right), K_{0} \stackrel{\$}{\leftarrow} \mathcal{K}$ and $b \stackrel{\$}{\leftarrow}\{0,1\}$

5) $\left(\tau\right.$, state $\left._{2}\right):=\mathcal{A}_{C C A 2}^{\mathcal{O}_{\text {Sym }}, \mathcal{O}_{\text {Encap }}, \mathcal{O}_{\text {Decap }}}\left(K_{b}\right.$, state $\left._{1}\right)$

6) $E:=\operatorname{Encap}(\varpi, \tau)$

7) $b^{\prime}:=\mathcal{A}_{C C A 2}^{\mathcal{O}_{\text {Sym }}, \mathcal{O}_{\text {Encap }}, \mathcal{O}_{\text {Decap }}}\left(E\right.$, state $\left._{2}\right)$

Fig. 3. IND-CCA2 game [60].

\begin{tabular}{|l|}
\hline SUF-CMA Game for SCTKEM: \\
\hline 1) $I:=\operatorname{Com}\left(1^{\lambda}\right)$ \\
2) $\left(S C S K_{s}, S C P K_{s}\right):=K e y G e n_{r}(I)$ \\
3) $\left(E, \tau, S C S K_{r}\right):=\mathcal{F}_{C M A}^{\mathcal{O}_{\text {Sym }}, \mathcal{O}_{\text {Encap }}, \mathcal{O}_{\text {Decap }}}\left(S C P K_{s}\right)$ \\
\hline
\end{tabular}

Fig. 4. SUF-CMA game [60].

a valid encapsulation $E$ from the sender to any receiver, with adaptive access to the three oracles. The adversary is allowed to come up with the presumed secret key $S C S K_{r}$ as part of his forgery [60]:

The adversary $\mathcal{F}_{C M A}$ wins the SUF-CMA game if

$$
\perp \neq \operatorname{Decap}\left(S C P K_{s}, S C S K_{r}, E, \tau\right)
$$

and the encapsulation oracle never returns $E$ when he queries on the tag $\tau$. The advantage of $\mathcal{F}_{C M A}$ is the probability that $\mathcal{F}_{C M A}$ wins the $S U F-C M A$ game. A signcryption tag-KEM is $S U F-C M A$ secure if the winning probability of the $S U F-C M A$ game by $\mathcal{F}_{C M A}$ is negligible.

Definition 1: A signcryption tag-KEM is said to be secure if it is IND-CCA2 and SUF-CMA secure.

\section{Generic security criteria of hybrid signcryption tag-KEM+DEM}

Security criteria for hybrid signcryption: The security of a hybrid signcryption tag-KEM+DEM depends on those of the underlying signcryption tag-KEM and DEM. However, it is important to note that in the standard model a signcryption tag-KEM is secure if it is both IND-CCA2 and SUF-CMA secure. Therefore, the generic security criteria for hybrid signcryption tag-KEM+DEM is given by the following theorem: 
Theorem 1: [60], [14] Let $H S C$ be a hybrid signcryption scheme constructed from a signcryption tag$\mathrm{KEM}$ and a DEM. If the signcryption tag-KEM is IND-CCA2 secure and the DEM is one-time secure, then $H S C$ is IND-CCA2 secure. Moreover, if the signcryption tag-KEM is SUF-CMA secure, then $H S C$ is also SUF-CMA secure.

\section{CODE-BASED HYBRID SIGNCRYPTION}

In this section, we first design a code-based signcryption tag-KEM scheme. Then we combine it with a one-time (OT) secure DEM for designing a hybrid signcryption tag-KEM+DEM scheme.

\section{A. Code-based signcryption tag-KEM scheme}

For designing our code-based signcryption tag-KEM scheme, we use the McEliece scheme as the underlying encryption scheme. More specifically, in order to achieve the CCA2 security for our schemes, we use McEliece's scheme with the Fujisaki-Okamoto conversion [33], [16]. The authors of [16] gave an instantiation of this scheme using generalized Srivastava (GS) codes. Indeed, by using GS codes, it seems possible to choose secure parameters even for codes defined over relatively small extension fields. However, Barelli and Couvreur recently introduced an efficient structural attack [6] against some of the candidates in the NIST post-quantum cryptography standardization process. Their attack is against codebased encryption schemes using some quasi-dyadic alternant codes with extension degree 2 . It works specifically for schemes based on GS code called DAGS [4]. Therefore, in our work we use the Goppa code with the Classic McEliece parameters. As for the underlying signature scheme, we use the code-based Wave [23] as described earlier.

The fact that we use Wave, the sender's secret key is a generalized $(U, U+V)$-code over a finite field $\mathbb{F}_{q}$ with $q>2$. Its public key is a parity-check matrix of a code equivalent to the previous one. To address the indistinguishability issue with high rate Goppa code, we use Goppa subcode equivalent for the receiver's public key. In Fig. 5, we describe the algorithm Com which will provide common parameters for our scheme.

Com
Input: $\left(1^{\lambda}\right)$
Output:
- Parameters of sender's generalized $(U, U+V)$-code: code length $n_{s}$,
dimension $k_{U}$ of $\mathrm{U}$, dimension $k_{V}$ of $\mathrm{V}$, dimension $k_{s}=k_{U}+k_{V}$ of
the generalized $(U, U+V)$-code, weight of error vector $\omega$, cardinality
$q$ of the finite field $\mathbb{F}_{q}$.
- Parameters of receiver's Goppa code: degree $m$ of extension $\mathbb{F}_{2^{m}}$ of
$\mathbb{F}_{2}$, length $n_{r}$ of the Goppa code, degree $t$ of Goppa polynomial $g_{r}$,
dimension $\tilde{k}$ of Goppa subcode.
- A cryptographic hash functions $\mathcal{H}_{1}:\{0,1\}^{*} \longrightarrow\{0,1\}^{\tilde{k}}$
- A cryptographic hash functions $\mathcal{H}_{0}:\{0,1\}^{*} \longrightarrow\{0,1\}^{\ell}$ where $\ell$ is the
bit length of the symmetric encryption key.
- A hash function $\mathcal{H}_{2}:\{0,1\}^{*} \longrightarrow\{0,1,2\}^{r_{s}}$ where $r_{s}=n_{s}-k_{s}$.
- A cryptographic hash function $\mathcal{H}_{3}:\{0,1\}^{*} \longrightarrow\{0,1\}^{\tilde{k}+\ell}$
- An encoding function $\phi: \mathbb{F}_{2}^{\kappa} \vec{\longrightarrow} \mathcal{W}_{2, n_{r}, t}$ where $\kappa$ is a well chosen
parameters such that $\kappa=\left|\left(\begin{array}{c}t \\ n_{r}\end{array}\right)\right|$ and $\mathcal{W}_{2, n_{r}, t}$ is the set of binary
vectors of length $n_{r}$ and Hamming weight $t$.

Fig. 5. Description of Common parameters. 
We give key generation algorithms in Figure 6, where we denote the sender key generation algorithm by KeyGenS and that of the receiver by KeyGenR. The receiver algorithm KeyGenR returns as signcryption public key a generator matrix $\mathbf{G}_{p k, r} \in \mathbb{F}_{2}^{\tilde{k} \times n_{r}}$ of a Goppa subcode equivalent. It returns as signcryption secret key the tuple $\left(g_{r}, \Gamma_{r}, \mathbf{S}_{r}^{-1}, \mathbf{P}_{r}\right)$, where $\Gamma_{r}$ and $g_{r}$ are, respectively, the support and the polynomial of a Goppa code. $\mathbf{S}_{r} \in \mathbb{F}_{2}^{\tilde{k} \times k_{r}}$ is a full rank matrix and $\mathbf{P}_{r}$ a permutation matrix. The sender key generation algorithm KeyGenS returns as private key three matrices $\mathbf{S}_{s} \in \mathbb{F}_{3}^{\left(n_{s}-k_{s}\right) \times\left(n_{s}-k_{s}\right)}, \mathbf{H}_{s k, s} \in \mathbb{F}_{3}^{\left(n_{s}-k_{s}\right) \times n_{s}}$ and $\mathbf{P}_{s} \in \mathbb{F}_{2}^{n_{s} \times n_{s}}$, where $\mathbf{S}_{s} \in \mathbb{F}_{3}^{\left(n_{s}-k_{s}\right) \times\left(n_{s}-k_{s}\right)}$ is an invertible matrix, $\mathbf{H}_{s k, s} \in \mathbb{F}_{3}^{\left(n_{s}-k_{s}\right) \times n_{s}}$ a parity-check matrix of a random generalized $(U, U+V)$-code and $\mathbf{P} \in \mathbb{F}_{2}^{n_{s} \times n_{s}}$ a permutation matrix. The sender public key is a parity-check matrix $\mathbf{H}_{p k, s} \in \mathbb{F}_{3}^{\left(n_{s}-k_{s}\right) \times n_{s}}$ of a generalized $(U, U+V)$ equivalent code given by $\mathbf{H}_{p k, s}=\mathbf{S}_{s} \mathbf{H}_{s k, s} \mathbf{P}_{s}$.

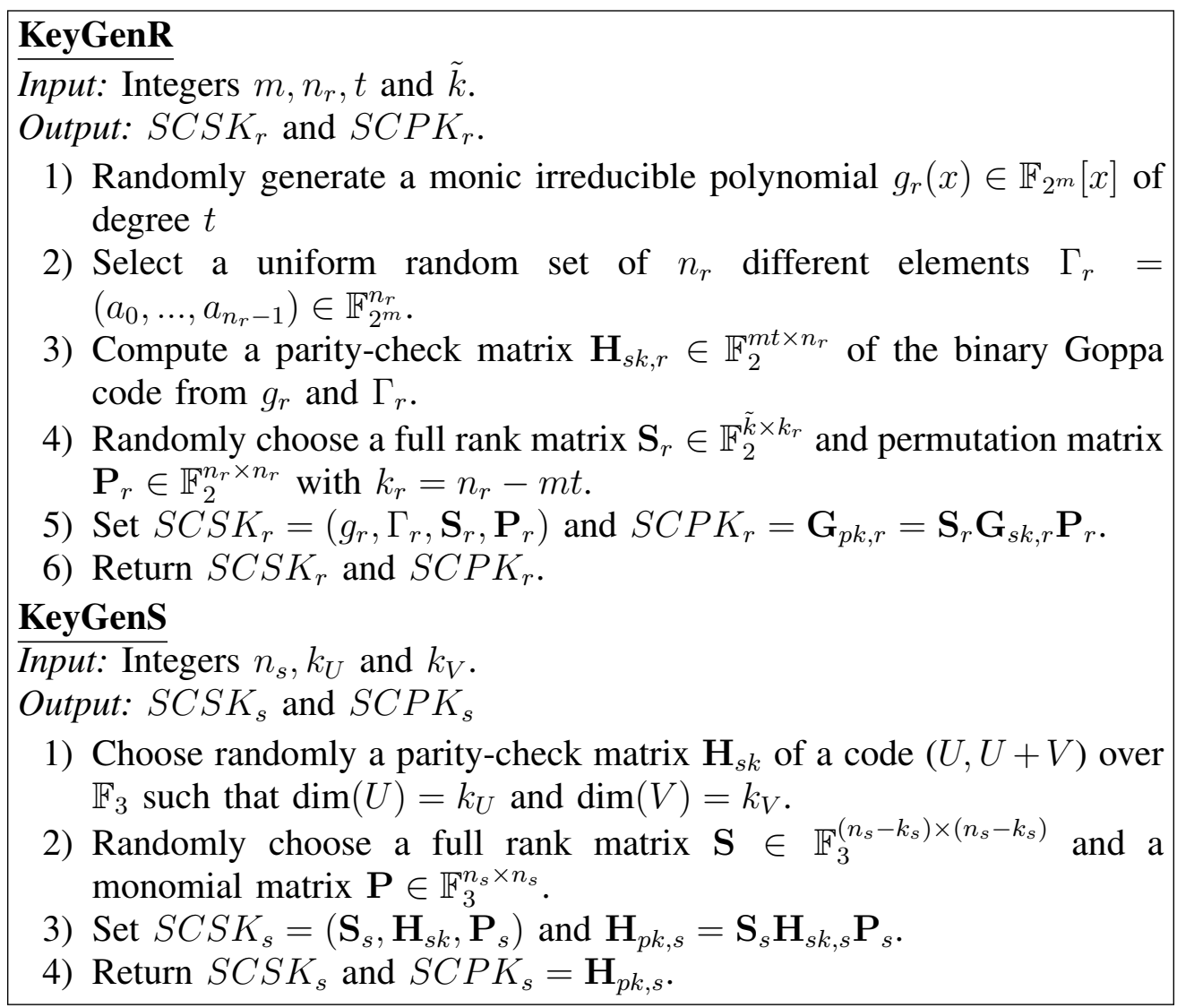

Fig. 6. Description of Common parameters and key generation algorithm.

In Figure 7, we give the design of the symmetric key generation algorithm Sym of our scheme. The algorithm Sym takes as input the bit length $\ell$ of the symmetric encryption key. It outputs an internal state information $\varpi$ and the session key $K$, where $\varpi$ is randomly chosen from $\mathbb{F}_{2}^{\ell}$, and $K$ is computed by using the hash function $\mathcal{H}_{0}$.

Figure 8 provides a description of the encapsulation and decapsulation algorithms of our signcryption tag-KEM scheme. We denote the encapsulation algorithm by Encap and the decapsulation by Decap. In the encapsulation algorithm, the sender first performs a particular Wave signature on the message $\boldsymbol{m}=\tau \| \varpi$, where $\varpi$ corresponds to an internal state information and $\tau$ is the input tag. The signature in the Wave scheme comprises two parts: an error vector $\boldsymbol{e} \in \mathbb{F}_{3}^{n_{s}}$ and a random binary vector $\boldsymbol{y}$. In our scheme, $\boldsymbol{z}$ is the hash of a random coin $\boldsymbol{y} \in \mathbb{F}_{2}^{\kappa}$. The sender then performs an encryption of $\boldsymbol{m}^{\prime}=\mathcal{H}_{1}(\tau) \| \varpi$. The encryption that we use in our scheme is the IND-CCA2 secure McEliece encryption scheme with the Fujisaki-Okamoto conversion introduced by Cayrel et al. [16]. During the encryption, the sender adaptively 


\section{Sym}

Input: The bit length $\ell$ of the symmetric encryption key.

Output: An internal state information $\varpi$ and a session key $K$.

1) $\boldsymbol{x} \stackrel{\$}{\leftarrow} \mathbb{F}_{2}^{\ell}$

2) Compute $K:=\mathcal{H}_{0}(\boldsymbol{x})$

3) Set $\varpi:=\boldsymbol{x}$

4) Return $(K, \varpi)$

Fig. 7. Description of the Sym algorithm.

uses the random binary vector $\boldsymbol{y}$ as a random coin. The resulting ciphertext is denoted by $\boldsymbol{c}$. The output is given by $E=(\boldsymbol{e}, \boldsymbol{c})$.

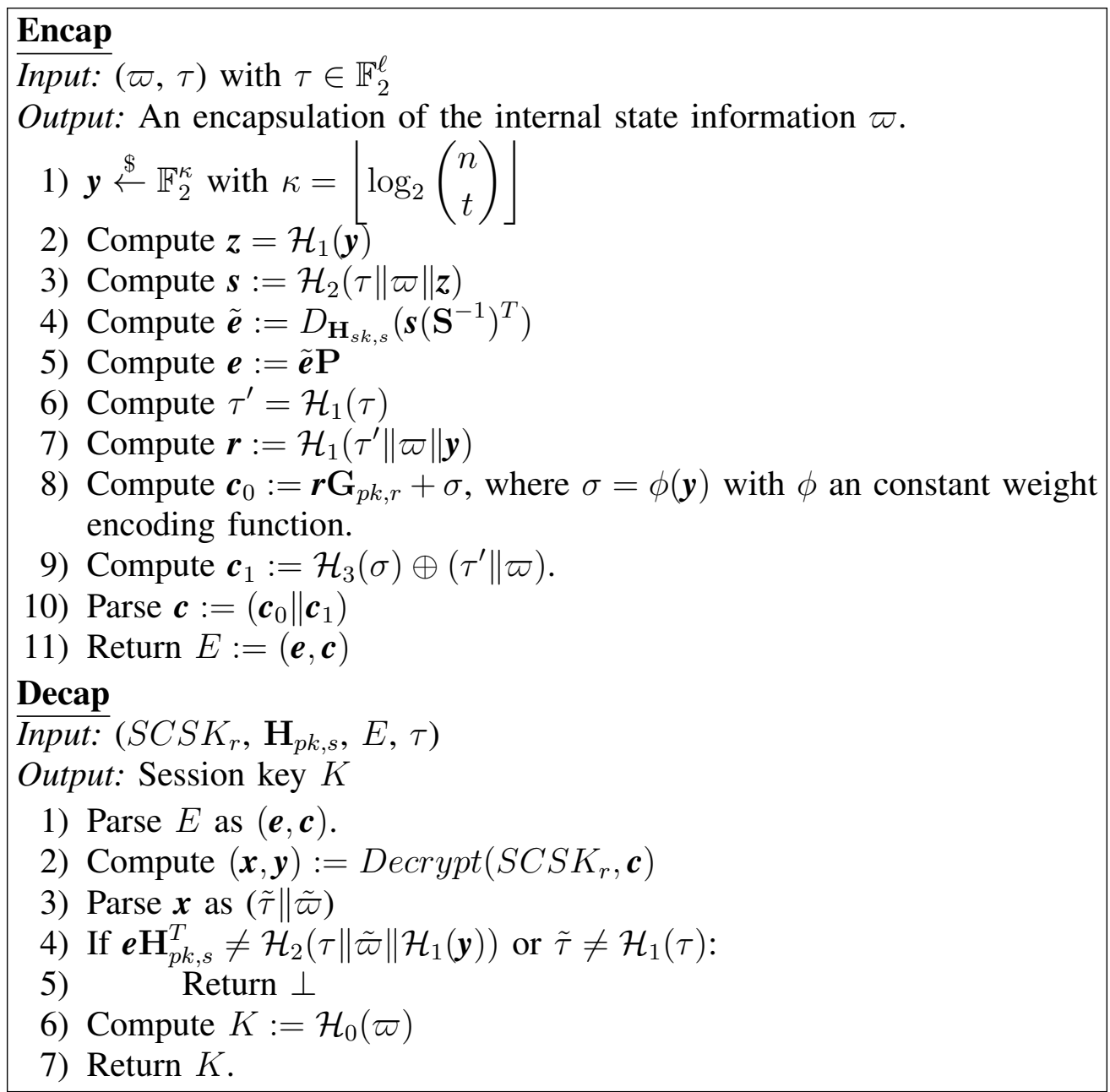

Fig. 8. Description of the Encap and Decap algorithms.

In the decapsulation algorithm Decap, the receiver first performs recovery of the internal state information $\varpi$ by using the algoritm Decrypt and the second part of the signature of $\boldsymbol{m}$. Then it verifies the signature and computes the session $K$ by using $\varpi$.

The algorithm Decrypt that we use in the decapsulation algorithm of our scheme is described in Figure 9. It is similar to that described in [16] but we introduce some modifications which are:

- we use an encoding function $\phi$ 
- the output is not only the clear message $\boldsymbol{m}$, but a pair $(\boldsymbol{m}, \boldsymbol{y})$ where $\boldsymbol{y}$ is the reciprocal image the error vector $\sigma$ by the encoding function $\phi$

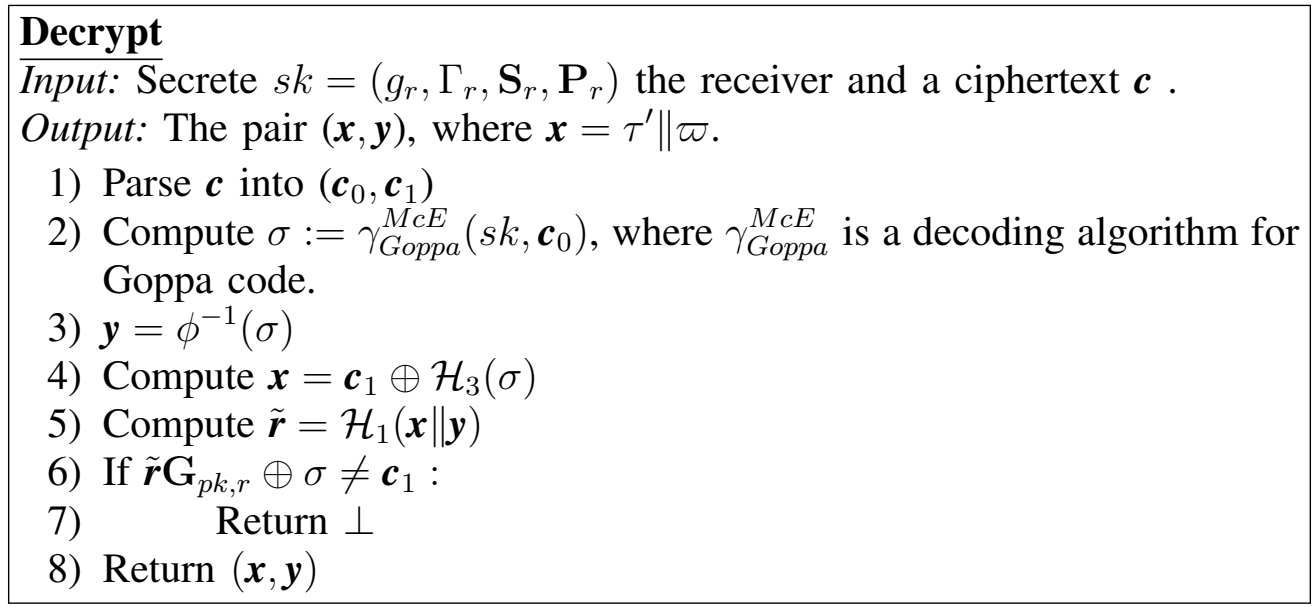

Fig. 9. Description of the Sym algorithm.

Completeness of our signcryption tag-KEM: Let $\tau$ be a tag, $\left(S C S K_{s}, S C P K_{s}\right)$ (resp. (SCSK $K_{r}$ and $\left.S C P K_{r}\right)$ ) be sender's (resp. receiver's) key pair generated by the algorithm KeyGen with input $1^{\lambda}$. Let $(K, \varpi):=\operatorname{Sym}\left(S C S K_{s}, S C P K_{r}\right)$ be a pair of a session key and an internal state information. Let $E:=(\boldsymbol{e}, \boldsymbol{c})$ be an encapsulation of the internal state information $\varpi$. Assuming that the encapsulation and decapsulation are performed by an honest user, we have:

- The receiver can recover the pair $\left(\tau^{\prime} \| \varpi, y\right)$ from $c$ and verify successfully that

$$
\boldsymbol{e} \mathbf{H}_{p k, s}^{T}=\mathcal{H}_{2}(\tau \| \varpi \mid \boldsymbol{y}) \text { and } \tau^{\prime}=\mathcal{H}_{1}(\tau)
$$

Otherwise the receiver performs a successful signature verification of message $\boldsymbol{m}:=\tau \| \varpi$ signed by an honest user using the dual version of mCFS signature.

- Therefore it can compute the session key $K:=\mathcal{H}_{0}(\varpi)$.

\section{B. Code-based hybrid signcryption}

Here we use the signcryption tag-KEM described in Section IV-A for designing a code-based hybrid signcryption. For the data encapsulation we propose the use of a regular OT-secure symmetric encryption scheme. We denote the symmetric encryption algorithm being used by SymEncrypt and the symmetric decryption algorithm by SymDecrypt.

Figure 10 gives the design of our code-based hybrid signcryption tag-KEM+DEM. In this design, algorithms Com, KeyGen and KeyGen are the same as those of our signcrytion tag-KEM. Algorithms Sym and Encaps are those of our signcryption tag-KEM in Section IV-A.

\section{SECURITY ANALYSIS}

Before discussing the security of our hybrid scheme, let us consider the following assumptions for our security analysis:

Assumption 1: The advantage of probabilistic polynomial-time algorithm $\mathcal{A}$ to solve the decoding random linear codes problem is negligible with respect to the length $n$ and dimension $k$ of the code.

Assumption 2: The advantage of probabilistic polynomial-time algorithm $\mathcal{A}$ to solve the $(U, U+V)$ distinguishing problem is negligible with respect to the length $n$ and dimension $k$ of the code.

Assumption 3: The advantage of probabilistic polynomial-time algorithm $\mathcal{A}$ to solve the subcode equivalence problem is negligible with respect to the length $n$ and dimension $k$ of the code. 


\section{Signcryption \\ Input: A three tuple $\left(\mathrm{SCSK}_{s}, \mathrm{SCPK}_{r}, \boldsymbol{m}\right)$ \\ Output: The signcrypted message $\boldsymbol{c}=(E, C)$. \\ 1) Compute $(K, \varpi)=\operatorname{Sym}\left(\mathrm{SCSK}_{s}, \mathrm{SCPK}_{r}\right)$ \\ 2) Compute $C=\operatorname{SymEncrypt}(K, \boldsymbol{m})$ \\ 3) Compute $E=\operatorname{Encaps}(\varpi, C)$ \\ 4) Return $(E, C)$ \\ Unsigncryption \\ Input: A three tuple $\left(\mathrm{SCSK}_{r}, \mathrm{SCPK}_{s},(\boldsymbol{c}, C)\right)$ \\ Output: The clear text $\boldsymbol{m}$ \\ 1) If Decaps $\left(\mathrm{SCSK}_{r}, \mathrm{SCPK}_{s}, \boldsymbol{c}\right)=\perp$ return $\perp$ \\ 2) Compute $\boldsymbol{m}=S y m D e c r y p t(K, C)$ \\ 3) Return $\boldsymbol{m}$}

Fig. 10. Code-based hybrid signcryption from SCTKEM and DEM.

Assumption 4: The advantage of probabilistic polynomial-time algorithm $\mathcal{A}$ to solve the decoding one out of many (DOOM) problem is negligible with respect to the length $n$ and dimension $k$ of the code.

\section{A. Information-set decoding algorithm}

In code-based cryptography, the best known non-structural attacks rely on information-set decoding. The information-set decoding algorithm was introduced by Prange [51] for decoding cyclic codes. After the publication of Prange's work, there have been several works studying to invert code-based encryption schemes based on information-set decoding (see [2] Section 4.1).

For a given linear code of length $n$ and dimension $k$, the main idea behind the information-set decoding algorithm is to find a set of $k$ coordinates of a garbled vector that are error-free and such that the restriction of the code's generator matrix to these positions is invertible. Then, the original message can be computed by multiplying the encrypted vector by the inverse of the submatrix.

Thus, those $k$ bits determine the codeword uniquely, and hence the set is called an information set. It is sometimes difficult to draw the exact resistance to this type of attacks. However, they are always lower-bounded by the ratio of information sets without errors to total possible information sets, i.e.,

$$
R_{I S D}=\frac{\left(\begin{array}{c}
n-\omega \\
k
\end{array}\right)}{\left(\begin{array}{l}
n \\
k
\end{array}\right)},
$$

where $\omega$ is the Hamming weight of the error vector. Therefore, a well chosen parameters can avoid these non-structural attacks. In our scheme, we use the parameters of the Wave signature [23] for the sender and those of Classic McEliece [2] for the receiver in the underlying encryption scheme.

\section{B. Key recovery attack}

In our case, the key recovery attack is at two different levels: the first one is on the sender side and the second one on the receiver side.

On the receiver side, it consists of the recovery of the Goppa polynomial $g_{r}$ and the support $\gamma_{r}=$ $\left(\alpha_{0}, \ldots, \alpha_{n-1}\right)$ from the public matrix. Therefore, the natural way for this is to perform a brute-force attack: one can determine the sequence $\left(\alpha_{0}, \ldots, \alpha_{n-1}\right)$ from $g_{r}$ and the set $\left\{\alpha_{0}, \ldots, \alpha_{n-1}\right\}$, or alternatively determine $g_{r}$ from $\left(\alpha_{0}, \ldots, \alpha_{n-1}\right)$. A good choice of parameters can avoid this attack for the irreducible Goppa code the number of choices of $g_{r}$ is given by

$$
\frac{1}{t} \sum_{d \mid t} \mu(d) q^{\frac{t}{d}} .
$$


By using the parameters of Classic McEliece, we can see that the complexity for performing a bruteforce attack to find Goppa polynomial is more than $2^{800}$ for the parameters proposed in [2]. It is also important to note that performing the recovery attack also implies solving an instance of subcode equivalence problem. According to Assumption 3, solving this problem is hard in the worst case. The best way to solve the subcode equivalence problem is to perform an exhaustive search. Another technique for this is to proceed by solving an algebraic system.

In the case of the sender, the key recovery attack consists of first solving the $(U, U+V)$ distinguishing problem for finite fields of cardinality $q=3$. Therefore under Assumption 3 and with a well chosen set of parameters this attack would fail.

\section{IND-CCA2 and SUF-CMA security}

In code-based cryptography, the main approach to a chosen-ciphertext attack against the McEliece encryption scheme consists of adding two errors to the received word. If the decryption succeeds, it means that the error vector in the resulting word has the same weight as the previous one. In our signcryption tag-KEM scheme, this implies either to recover the session key $K$ or distinguish encapsulation of two different session keys from $(\boldsymbol{e}, \boldsymbol{c}, \tau)$. We see that the recovery of the session key $K$ corresponds to recovery of a plaintext in a CCA2 secure version of McEliece's cryptosystem (see [16] Subsection 3.2). We now have the following theorem:

Theorem 2: Under Assumptions 1 and 3, the signcryption tag-KEM scheme described in Subsection IV-A is IND-CCA2 secure.

Proof: Let $\mathcal{A}_{C C A 2}$ be a PPT adversary against the signcryption tag-KEM scheme described in Subsection IV-A in the signcryption tag-KEM IND-CCA2 game. Let us denote its advantage by $\epsilon_{C C A 2, S c T k e m}$. For proving Theorem 2 we need to bound $\epsilon_{C C A 2, S c T k e m}$.

Game 0: This game is the normal signcryption tag-KEM IND-CCA2 game. Let us denote by $X_{0}$ the event that the adversary wins Game 0 and $\operatorname{Pr}\left(X_{0}\right)$ the probability that it happens. Then we have

$$
\operatorname{Pr}\left(X_{0}\right)=\epsilon_{C C A 2, \text { ScTkem }}
$$

Game 1: This game corresponds to the simulation of hash function oracle. Indeed it is the same as Game 0 except that adversary can have access to hash function oracle: It looks for some pair $\left(\tau^{*}, \boldsymbol{y}^{*}\right) \in \mathbb{F}_{2}^{\lambda} \times \mathbb{F}_{2}^{\kappa}$ such that $\boldsymbol{e} \mathbf{H}_{s}^{T}=\mathcal{H}_{2}\left(\tau^{*}\|\varpi\| \mathcal{H}_{1}\left(\boldsymbol{y}^{*}\right)\right)$. Then, it tries to continue by computing $\boldsymbol{c}^{\prime}$. We can see that it could succeed at least when the following collisions happen:

$$
\mathcal{H}_{1}\left(\tau^{*}\right)=\mathcal{H}_{1}(\tau) \text { and } \mathcal{H}_{1}\left(\tau^{*}\|\varpi\| \mathcal{H}_{2}\left(\boldsymbol{y}^{*}\right)\right)=\mathcal{H}_{2}\left(\tau\|\varpi\| \mathcal{H}_{1}(\boldsymbol{y})\right)
$$

Therefore, if $q_{h}$ is the number of queries allowed and $X_{1}$ the event that $\mathcal{A}_{C C A 2}$ wins game $X_{1}$, then we have:

$$
\left|\operatorname{Pr}\left(X_{0}\right)-\operatorname{Pr}\left(X_{1}\right)\right| \leq \frac{q_{h}}{\left(\begin{array}{c}
n \\
t
\end{array}\right)}
$$

Game 2: This game is the same as Game 1 except that the error vector $\boldsymbol{e}$ in the encapsulation output is generated randomly. We can see that the best to proceed is to split $\boldsymbol{c}$ as $\left(\boldsymbol{c}_{0} \| \boldsymbol{c}_{1}\right)$ and then try to invert either $\boldsymbol{c}_{0}$ for recovering the error $\sigma$ or $\boldsymbol{c}_{1}$ for recovering directly the internal state $\varpi_{b}$. That means that the adversary is able either to solve the syndrome decoding problem or to invert an one-time pad function. Therefore we have:

$$
\left|\operatorname{Pr}\left(X_{1}\right)-\operatorname{Pr}\left(X_{2}\right)\right| \leq \epsilon_{S D}+\nu(\ell)
$$

where $\epsilon_{S D}$ is the advantage of an adversary against the syndrome decoding problem, $\nu$ a negligible function and $\ell$ the bit length of the symmetric encryption.

We can show that if the adversary $\mathcal{A}_{C C A 2}$ wins this game, we can use it to construct an adversary $\mathcal{A}_{M c E}$ for attacking the underlying McEliece scheme in the public key encryption IND-CCA2 game 
(called PKE.Game in Appendix A). For more details on the underlying McEliece encryption scheme and its IND-CCA2 security proof, the reader is referred to Appendix C. We now proceed as follows:

- Given the receiver public key $p k$ which corresponds to a receiver public key signcryption tag-KEM, $\mathcal{A}_{M c E}$ :

$\star$ chooses randomly $\left(\varpi_{0}, \varpi_{1}\right) \stackrel{\$}{\leftarrow} \mathbb{F}_{2}^{\ell}$

$\star$ chooses randomly $\delta \stackrel{\$}{\leftarrow}\{0,1\}$

$\star$ sends the public key $p k$ and $\varpi_{\delta}$ to $\mathcal{A}_{C C A 2}$

- Given a tag $\tau$ from $\mathcal{A}_{C C A 2}, \mathcal{A}_{M c E}$ :

$\star$ sends the pair $\left(\mathcal{H}_{1}(\tau)\left\|\varpi_{0}, \mathcal{H}_{1}(\tau)\right\| \varpi_{1}\right)$ to the encryption oracle of PKE.Game

$\star$ forwards $c$ received from the encryption oracle to $\mathcal{A}_{C C A 2}$

- For every decryption query $\left(\boldsymbol{c}_{i}, \tau_{i}\right)$ from $\mathcal{A}_{C C A 2}$ :

$\star$ if $\boldsymbol{c}_{i}=\boldsymbol{c}, \mathcal{A}_{M c E}$ return $\perp$ to $\mathcal{A}_{C C A 2}$

$\star$ Otherwise it sends $\boldsymbol{c}_{i}$ to the decryption oracle of PKE.Game. Receiving $\tau_{i}^{\prime} \| \varpi_{i}$ from the decryption oracle:

$\triangleright$ if $\tau_{i}^{\prime} \neq \mathcal{H}_{1}\left(\tau_{i}\right)$, it returns $\perp$ to $\mathcal{A}_{C C A 2}$

$\triangleright$ Otherwise, it returns $\varpi_{i}$ to $\mathcal{A}_{C C A 2}$

- When $\mathcal{A}_{C C A 2}$ outputs $\tilde{\delta}=\delta, \mathcal{A}_{M c E}$ returns 1 . Otherwise, it returns 0 .

Let $\epsilon_{p k e}$ be the advantage of $\mathcal{A}_{M c E}$ in the PKE.Game. Note that the target ciphertext $c$ can be uniquely decrypted to $\left.\mathcal{H}_{1}(\tau)\right) \| \varpi_{\delta}$. Therefore any $\left(\boldsymbol{c}, \tau^{\prime}\right)$ other than $(\boldsymbol{c}, \tau)$ can not be a valid signcryption ciphertext unless a collusion of $\mathcal{H}_{1}$ takes place, i.e., $\mathcal{H}_{1}\left(\tau_{i}\right)=\mathcal{H}_{1}(\tau)$. The correct answer to any decryption query with $\boldsymbol{c}_{i}=\boldsymbol{c}$ is $\perp$. Decryption queries from $\mathcal{A}_{C C A 2}$ are correctly answered since $\boldsymbol{c}_{i}$ is decrypted by the decryption oracle of PKE.Game.

When $\mathcal{A}_{C C A 2}$ outputs $\tilde{\delta}$, it means that $\varpi_{\delta}$ is embedded in $\boldsymbol{c}_{i}$ otherwise $\varpi_{1-\delta}$ is embedded. It means that the adversary $\mathcal{A}_{M c E}$ wins game PKE.Game with the same probability as $\mathcal{A}_{C C A 2}$ wins Game 2 when collision of $\mathcal{H}_{1}$ has happened. Let $\tilde{X}$ be the event collision of $\mathcal{H}_{1}$ has happened and $\tilde{X}_{2}$ the event $\mathcal{A}_{M c E}$ wins the PKE.Game. Let us denote by $\epsilon_{p k e}$ the probability of the event $\tilde{X}_{2}$ and $\epsilon_{c o l}$ that of $\tilde{X}$. Therefore we have:

$$
\operatorname{Pr}\left(X_{2} \mid \tilde{X}\right)=\operatorname{Pr}\left(\tilde{X}_{2}\right) \Longrightarrow \operatorname{Pr}\left(X_{2}\right) \leq \operatorname{Pr}\left(\tilde{X}_{2}\right)+\operatorname{Pr}(\tilde{X})
$$

By putting it all together, we conclude our proof.

Theorem 3: Under Assumptions 3 and 4, the signcryption tag-KEM scheme described in Subsection IV-A is SUF-CMA secure.

Proof: Let $\mathcal{F}_{C M A}$ be an adversary against our signcryption tag-KEM in the SUF-CMA game and $\epsilon_{C M A}$ its advantage. For the forgery of our signcryption, adversary $\mathcal{F}_{C M A}$ needs to first find a pair $(\boldsymbol{e}, \boldsymbol{y}) \in \mathcal{W}_{q, n, \omega} \times \mathbb{F}_{2}^{\tilde{k}}$ such that $\boldsymbol{e} \mathbf{H}_{p k, s}^{T}=\mathcal{H}_{2}(\tau\|\varpi\| \boldsymbol{y})$. Then, it will try to find $\boldsymbol{r} \in \mathbb{F}_{2}^{\kappa}$ such that $\mathcal{H}_{1}(\boldsymbol{r})=\boldsymbol{y}$, i.e., it wins in the target pre-image free game (see Appendix B) against the cryptographic hash function $\mathcal{H}_{1}$. We can see that finding $(\boldsymbol{e}, \boldsymbol{y}) \in \mathcal{W}_{q, n, \omega} \times \mathbb{F}_{2}^{\tilde{k}}$ such that $\boldsymbol{e} \mathbf{H}_{p k, s}^{T}=\mathcal{H}_{2}(\tau\|\varpi\| \boldsymbol{y})$ corresponds to the forgery of the underlying Wave signature scheme. Let $\epsilon_{\text {PreIm }}$ be the advantage of an adversary in the pre-image free game against a cryptographic hash function. Let $\mathcal{A}_{W a v e, C M A}$ be an adversary against the Wave signature in the EUF-CMA game and $\epsilon_{W a v e, E U F}$ its advantage. Let $X$ be the event that $\mathcal{A}_{W a v e, C M A}$ wins. Let $\tilde{X}$ be the event that adversary is able to find a pre-image $\boldsymbol{x}$ of $\boldsymbol{y}$ by $\mathcal{H}_{1}$ such that $\boldsymbol{x} \in \mathbb{F}_{2}^{\kappa}$. We have:

$$
\begin{aligned}
\operatorname{Pr}\left(\mathcal{F}_{C M A} \text { wins }\right) & =\operatorname{Pr}(X \text { and } \tilde{X}) \\
\leq & \operatorname{Pr}(X)+\operatorname{Pr}(\tilde{X}) \\
\leq & \epsilon_{\text {Wave } E U F}+\frac{\epsilon_{\text {PreIm }}}{2^{\kappa}}
\end{aligned}
$$

Note that due to the fact that $\mathcal{H}_{1}$ is a cryptographic hash function, $\epsilon_{\text {PreIm }}$ is negligible and that concludes our proof. 
Corollary 1: The signcryption tag-KEM described in Subsection IV-A is secure.

The above corollary is a consequence of Theorems 2 and 3 . We then have the following.

Theorem 4: Under Assumptions 1-4, the hybrid signcryption tag-KEM+DEM scheme described in Subsection IV-B is IND-CCA2 and SUF-CMA secure.

Proof: Under Assumptions 1-3, Theorems 2 and 3 provide mandatory conditions regarding signcryption tag-KEM for a secure hybrid signcryption (see Theorem 1). In addition, the symmetric encryption scheme used is OT-secure and hence a direct application of Theorem 1 allows us to achieve a proof of the theorem.

\section{PARAMETER VALUES}

For our scheme we choose parameters such that $\lambda_{0}=\lambda+2 \log _{2}\left(q_{\text {sign }}\right)$ and $\lambda_{M c E}$ of the underlying Wave signature and McEliece's encryption, respectively, satisfy $\max \left(\lambda_{0}, \lambda_{M c E}\right) \leq\left\lfloor\left(\begin{array}{c}n_{r} \\ t\end{array}\right)\right\rfloor$. According to the sender and receiver keys, the size of our ciphertext is given by

$$
|E|=|\boldsymbol{e}|+|\boldsymbol{c}|+|C|=2 n_{s}+n_{r}+\tilde{k}+2 \ell .
$$

Table I gives suggested values of the parameters of our scheme. These values have been derived using those of Wave [5] and Classic McEliece [2] for security Level 1 of NIST. According to the values given in Table I, the ciphertext size in bits of our scheme is in the order of $|E|=2.9 \times 10^{4}$.

\begin{tabular}{l|c|c|c|c|c|c|c|c|c} 
Parameter & $n_{s}$ & $k_{U}$ & $k_{V}$ & $\omega$ & $m$ & $t$ & $n_{r}$ & $\tilde{k}$ & $\ell$ \\
\hline Value & 8492 & 3558 & 2047 & 7980 & 12 & 64 & 3488 & 1815 & 512 \\
TABLE I \\
PARAMETER VALUES OF THE PROPOSED SCHEME.
\end{tabular}

Table II provides key sizes of our scheme in terms of relevant parameters. Then in Table III] we give a numerical comparison of key and ciphertext sizes of our scheme with some existing lattice-based hybrid signcryption schemes. The rationale behind comparing our scheme against lattice-based schemes is that no code-based hybrid signcryption scheme exists in the literature and the underlying hard problems in both code- and lattice-based schemes are considered quantum-safe. For the lattice based schemes in our comparison, the parameters, including plaintext size of 512 bits, are from [53, Table 2]. We can see that for post-quantum security level 1 the proposed scheme has the smallest key and ciphertext sizes.

\begin{tabular}{l|c|c} 
User & Public key & Secret key \\
\hline Receiver's key size & $\tilde{k} n_{r}$ & $m\left(2 n_{r}+t-\tilde{k} t\right)+\tilde{k} n_{r}$ \\
\hline Sender's key size & $r\left(n_{s}-r\right) \log _{2}(q)$ & $\left(n_{s}\left(n_{s}+r\right)+r^{2}\right) \log _{2}(q)$
\end{tabular}

TABLE II

KEY SIZES OF THE PROPOSED SCHEME.

\begin{tabular}{l|c|c|c|c|c}
\multirow{2}{*}{ Construction } & \multicolumn{2}{|c|}{ Receiver's key size } & \multicolumn{2}{c|}{ Sender's key size } & \multirow{2}{*}{ Ciph. size } \\
\cline { 2 - 5 } & Pub. key & Sec. key & Pub. key & Sec. key & \\
\hline$S C_{T K}$ [53], [19] & $8.5 \times 10^{7}$ & $4.2 \times 10^{8}$ & $8.4 \times 10^{7}$ & $4.2 \times 10^{8}$ & $5.5 \times 10^{5}$ \\
\hline$S C_{K E M}$ [53], [19] & $5.7 \times 10^{7}$ & $4.2 \times 10^{8}$ & $8.5 \times 10^{7}$ & $4.2 \times 10^{8}$ & $5.2 \times 10^{5}$ \\
\hline$S C_{C H K}$ [53], [46] & $2.8 \times 10^{7}$ & $4.2 \times 10^{8}$ & $2.8 \times 10^{7}$ & $4.2 \times 10^{8}$ & $4.5 \times 10^{6}$ \\
\hline Shingo and Junji [53] & $2.8 \times 10^{7}$ & $4.2 \times 10^{8}$ & $2.8 \times 10^{7}$ & $4.2 \times 10^{8}$ & $4.0 \times 10^{5}$ \\
\hline Our scheme & $6.3 \times 10^{6}$ & $5.0 \times 10^{6}$ & $2.6 \times 10^{7}$ & $1.7 \times 10^{8}$ & $2.1 \times 10^{4}$
\end{tabular}

TABLE III

SIZE COMPARISON (IN BITS) OF THE PROPOSED SCHEME WITH THE LATTICE BASED SCHEMES OF [53], [46], [19]. 


\section{CONCLUSION}

In this paper, we have proposed a new signcryption tag-KEM based on coding theory. The security of our scheme relies on known hard problems in coding theory. We have used the proposed signcryption schemed to design a new code-based hybrid signcryption tag-KEM+DEM. We have proven that the proposed schemes are IND-CCA2 and SUF-CMA secure against any probabilistic polynomial-time adversary. The proposed scheme has a smaller ciphertext size compared to the pertinent lattice-based schemes.

\section{REFERENCES}

[1] N. Aragon, O. Blazy, P. Gaborit, A. Hauteville, and G. Zémor, "Durandal: a rank metric based signature scheme," in Annual International Conference on the Theory and Applications of Cryptographic Techniques. Springer, 2019, pp. 728-758. 55

[2] M. R. Albrecht, D. J. Bernstein et al., "Classic McEliece: conservative code-based cryptography." Online Available: https://classic. mceliece.org/nist/mceliece-20201010.pdf $12,13,15$

[3] J. Baek, R. Steinfeld, and Y. Zheng, "Formal proofs for the security of signcryption," Journal of Cryptology, vol. 20, no. 2, pp. 203-235, 2007. 6

[4] G. Banegas, P. S. Barreto, B. O. Boidje, P.-L. Cayrel, G. N. Dione, K. Gaj, C. T. Gueye, R. Haeussler, J. B. Klamti, O. N'diaye et al., "Dags: Key encapsulation using dyadic gs codes," Journal of Mathematical Cryptology, vol. 12, no. 4, pp. 221-239, 2018. 2 , 8

[5] G. Banegas, T. Debris-Alazard, M. Nedeljkovic, and B. Smith, "Wavelet: Code-based postquantum signatures with fast verification on ' microcontrollers," arXiv preprint arXiv:2110.13488, 2021. 5. 15

[6] E. Barelli and A. Couvreur, "An efficient structural attack on nist submission dags," in International Conference on the Theory and Application of Cryptology and Information Security. Springer, 2018, pp. 93-118 8

[7] P. S. Barreto, B. Libert, N. McCullagh, and J.-J. Quisquater, "Signcryption schemes based on the Diffie-Hellman problem," in Practical Signcryption. Springer, 2010, pp. 57-69. 1

[8] P. S. Barreto, B. Libert, N. McCullagh and J. Quisquater, "Signcryption schemes based on bilinear maps," in Practical Signcryption. Springer, 2010, pp. 71-97. 1

[9] P. S. Barreto, R. Lindner, and R. Misoczki, "Monoidic codes in cryptography," in International Workshop on Post-Quantum Cryptography. Springer, 2011, pp. 179-199. 3

[10] T. P. Berger, C. T. Gueye, and J. B. Klamti, "A NP-complete problem in coding theory with application to code based cryptography," in International Conference on Codes, Cryptology, and Information Security. Springer, 2017, pp. 230-237. 23

[11] T. P. Berger and P. Loidreau, "How to mask the structure of codes for a cryptographic use," Designs, Codes and Cryptography, vol. 35, no. 1 , pp. 63-79, 2005. 3

[12] T. P. Berger, P.-L. Cayrel, P. Gaborit, and A. Otmani, "Reducing key length of the McEliece cryptosystem," in International Conference on Cryptology in Africa. Springer, 2009, pp. 77-97. 3

[13] E. Berlekamp, R. McEliece, and H. Van Tilborg, "On the inherent intractability of certain coding problems (corresp.)," IEEE Transactions on Information Theory, vol. 24, no. 3, pp. 384-386, 1978. 3

[14] T. E. Bjørstad and A. W. Dent, "Building better signcryption schemes with tag-KEMs," in International Workshop on Public Key Cryptography. Springer, 2006, pp. 491-507. 16

[15] J.-F. Biasse, G. Micheli, E. Persichetti, and P. Santini, "Less is more: Code-based signatures without syndromes." IACR Cryptol. ePrint Arch., vol. 2020, p. 594, 2020. 5

[16] P.-L. Cayrel, G. Hoffmann, and E. Persichetti, "Efficient implementation of a CCA2-secure variant of McEliece using generalized Srivastava codes," in International Workshop on Public Key Cryptography. Springer, 2012, pp. 138-155. 2, 3, 8, 9, 10, 18

[17] P.-L. Cayrel, C. T. Gueye, O. Ndiaye, E. Persichetti et al., "Efficient implementation of hybrid encryption from coding theory," in International Conference on Codes, Cryptology, and Information Security. Springer, 2017, pp. 254-264. 4

[18] P.-L. Cayrel, P. Véron, and S. M. E. Y. Alaoui, "A zero-knowledge identification scheme based on the q-ary syndrome decoding problem," in International Workshop on Selected Areas in Cryptography. Springer, 2010, pp. 171-186. 5

[19] D. Chiba, T. Matsuda, J. C. Schuldt, and K. Matsuura, "Efficient generic constructions of signcryption with insider security in the multi-user setting," in International Conference on Applied Cryptography and Network Security. Springer, 2011, pp. 220-237. 15

[20] N. T. Courtois, M. Finiasz, and N. Sendrier, "How to achieve a McEliece-based digital signature scheme," in International Conference on the Theory and Application of Cryptology and Information Security. Springer, 2001, pp. 157-174. 5

[21] R. Cramer and V. Shoup, "Design and analysis of practical public-key encryption schemes secure against adaptive chosen ciphertext attack," SIAM Journal on Computing, vol. 33, no. 1, pp. 167-226, 2003. 4

[22] T. Debris-Alazard, N. Sendrier, and J.-P. Tillich, "The problem with the surf scheme," arXiv preprint arXiv:1706.08065, 2017. 3

[23] T. Debris-Alazard, N. Sendrier, and J.-P. Tillich, "Wave: A new code-based signature scheme," Cryptology ePrint Archive: Report 2018/996, 2018, https://eprint.iacr.org/2018/996/20181022:154324 5 , 12

[24] T. Debris-Alazard, N. Sendrier, and J.-P. Tillich, "Wave: A new family of trapdoor one-way preimage sampleable functions based on codes," in International Conference on the Theory and Application of Cryptology and Information Security. Springer, 2019, pp. 21-51. 5

[25] A. W. Dent, "Hybrid cryptography," Cryptology ePrint Archive, Report 2004/210, 2004, https://eprint.iacr.org/2004/210 [1

[26] A. W. Dent, "Hybrid signcryption schemes with insider security," in Australasian Conference on Information Security and Privacy. Springer, 2005, pp. 253-266. 1

[27] A. W. Dent, "Hybrid signcryption schemes with outsider security," in International Conference on Information Security. Springer, 2005, pp. 203-217. 1 
[28] A. W. Dent and J. Malone-Lee, "Signcryption schemes based on the RSA problem," in Practical Signcryption. Springer, 2010, pp. 99-117. 1

[29] A. W. Dent and Y. Zheng, Eds., Practical signcryption, ser. Information Security and Cryptography. Springer, 2010.6

[30] J.-C. Faugere, V. Gauthier-Umana, A. Otmani, L. Perret, and J.-P. Tillich, "A distinguisher for high-rate McEliece cryptosystems," IEEE Transactions on Information Theory, vol. 59, no. 10, pp. 6830-6844, 2013. 3

[31] A. Fiat and A. Shamir, "How to prove yourself: Practical solutions to identification and signature problems," in Conference on the theory and application of cryptographic techniques. Springer, 1986, pp. 186-194. 5

[32] K. Fukushima, P. S. Roy, R. Xu, S. Kiyomoto, K. Morozov, and T. Takagi, "Racoss: Random code-based signature scheme,” Submission to NIST post-quantum standardization process, 2017. 5

[33] E. Fujisaki and T. Okamoto, "Secure integration of asymmetric and symmetric encryption schemes," in Annual International Cryptology Conference. Springer, 1999, pp. 537-554. 819

[34] A. Jain, S. Krenn, K. Pietrzak, and A. Tentes, "Commitments and efficient zero-knowledge proofs from learning parity with noise," in International Conference on the Theory and Application of Cryptology and Information Security. Springer, 2012, pp. 663-680. 5

[35] T. Johansson and F. Jonsson, "On the complexity of some cryptographic problems based on the general decoding problem," IEEE Transactions on Information Theory, vol. 48, no. 10, pp. 2669-2678, 2002. 3

[36] H. Q. Le, D. H. Duong, P. S. Roy, W. Susilo, K. Fukushima, S. Kiyomoto, "Lattice-based signcryption with equality test in standard model." Computer Standards \& Interfaces p.103515 (2021). 1

[37] F. Li, F. T. Bin Muhaya, M. K. Khan, and T. Takagi, "Lattice-based signcryption," Concurrency and Computation: Practice and Experience, vol. 25, no. 14, pp. 2112-2122, 2013. 1

[38] V. Lyubashevsky, "Fiat-shamir with aborts: Applications to lattice and factoring-based signatures," in International Conference on the Theory and Application of Cryptology and Information Security. Springer, 2009, pp. 598-616. 5

[39] Z. Li, C. Xing, and S. L. Yeo, "A new code based signature scheme without trapdoors." IACR Cryptol. ePrint Arch., vol. 2020, p. 1250, 2020. 5

[40] K. P. Mathew, S. Vasant, and C. P. Rangan, "On provably secure code-based signature and signcryption scheme," IACR Cryptology ePrint Archive, vol. 2012, p. 585, 2012. 2

[41] K. P. Mathew, S. Vasant, and C. P. Rangan, "Efficient code-based hybrid and deterministic encryptions in the standard model," in International Conference on Information Security and Cryptology. Springer, 2013, pp. 517-535. 4

[42] R. J. McEliece, "A public-key cryptosystem based on algebraic coding theory," DSN progress report, pp. 42-44, 1978. 3

[43] R. Misoczki and P. S. Barreto, "Compact McEliece keys from Goppa codes," in International Workshop on Selected Areas in Cryptography. Springer, 2009, pp. 376-392. 3

[44] R. Misoczki, J.-P. Tillich, N. Sendrier, and P. S. Barreto, "MDPC-McEliece: New McEliece variants from moderate density parity-check codes," in 2013 IEEE international symposium on information theory. IEEE, 2013, pp. 2069-2073. 3

[45] H. Niederreiter, "Knapsack-type cryptosystems and algebraic coding theory," Prob. Control and Inf. Theory, vol. 15, no. 2, pp. 159-166, 1986. 3

[46] R. Nakano and J. Shikata, "Constructions of signcryption in the multi-user setting from identity-based encryption," in IMA International Conference on Cryptography and Coding. Springer, 2013, pp. 324-343. 15

[47] E. Persichetti, "Compact McEliece keys based on quasi-dyadic Srivastava codes," Journal of Mathematical Cryptology, vol. 6, no. 2, pp. 149-169, 2012. 3

[48] E. Persichetti, "Secure and anonymous hybrid encryption from coding theory," in International Workshop on Post-Quantum Cryptography. Springer, 2013, pp. 174-187. 4

[49] E. Persichetti, "Efficient one-time signatures from quasi-cyclic codes: A full treatment," Cryptography, vol. 2, no. 4, p. 30, 2018. 5

[50] E. Persichetti, "Improving the efficiency of code-based cryptography," Ph.D. dissertation, University of Auckland, 2012. 5

[51] E. Prange, "The use of information sets in decoding cyclic codes," IRE Transactions on Information Theory, vol. 8, no. 5, pp. 5-9, 1962. 12

[52] N. Sendrier, "Decoding one out of many," in International Workshop on Post-Quantum Cryptography. Springer, 2011, pp. 51-67. 3

[53] S. Sato and J. Shikata, "Lattice-based signcryption without random oracles," in International Conference on Post-Quantum Cryptography. Springer, 2018, pp. 331-351. 1] 15

[54] Y. Song, Z. Li, Y. Li, and J. Li, "Attribute-based signcryption scheme based on linear codes," Information Sciences, vol. 417, pp. 301-309, 2017. 2

[55] Y. Song, X. Huang, Y. Mu, W. Wu, and H. Wang, "A code-based signature scheme from the lyubashevsky framework," Theoretical Computer Science, vol. 835, pp. 15-30, 2020. 5

[56] R. Steinfeld and Y. Zheng, "A signcryption scheme based on integer factorization," in International Workshop on Information Security. Springer, 2000, pp. 308-322. 1 .

[57] J. Stern, "A new identification scheme based on syndrome decoding," in Annual International Cryptology Conference. Springer, 1993, pp. 13-21. 5

[58] X. Yang, H. Cao, W. Li, and H. Xuan, "Improved lattice-based signcryption in the standard model," IEEE Access, vol. 7, pp. 155 552-155 562, 2019. 1

[59] J. Yan, L. Wang, L. Wang, Y. Yang, W. Yao, "Efficient lattice-based signcryption in standard model," Mathematical Problems in Engineering (2013). 1

[60] M. Yoshida and T. Fujiwara, "On the security of tag-KEM for signcryption," Electronic Notes in Theoretical Computer Science, vol. 171, no. 1, pp. 83-91, 2007. 1, 6, 7

[61] X. Zhao, X. Wang, "An efficient identity-based signcryption from lattice," International Journal of Security and Its Applications8(2), 363-374 (2014). 1

[62] Y. Zheng, "Digital signcryption or how to achieve cost (signature \& encryption)« cost (signature)+ cost (encryption)," in Annual international cryptology conference. Springer, 1997, pp. 165-179. 1 
[63] Y. Zheng and H. Imai, "How to construct efficient signcryption schemes on elliptic curves," Information processing letters, vol. 68, no. 5 , pp. 227-233, 1998

1

\section{APPENDIX}

\section{A. PKE.Game}

Here we recall the IND-CCA2 game for PKE called PKE.Game in our scheme. The decryption oracle is denoted by $\mathcal{O}$.

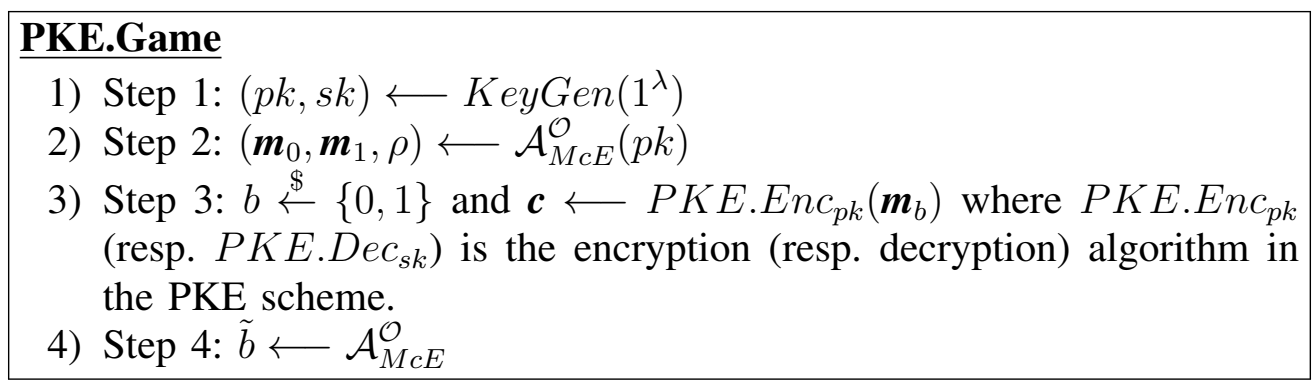

Fig. 11. PKE.Game

In Step 4. the adversary $\mathcal{A}_{M c E}$ is restricted not to make request to $\mathcal{O}$ on the ciphertext $\boldsymbol{c}$. Clear texts $\boldsymbol{m}_{0}$ and $\boldsymbol{m}_{1}$ must have the same length. $\mathcal{A}_{M c E}$ wins when $\tilde{b}=b$ and its advantage corresponds to the probability that it wins this game which is denoted by $\epsilon_{p k e}$.

\section{B. Target Preimage-Free}

Target Preimage-Free function is a special case of universal one-way function; An adversary is given $(\mathcal{H}, \boldsymbol{y})$ (chosen at random in their domain) and then attempts to find $\boldsymbol{x}$ such that $\mathcal{H}(\boldsymbol{x})=\boldsymbol{y}$. Let $\chi_{\lambda}=\{X\}$ be a collection of domains and $\chi=\left\{\chi_{\lambda}\right\}_{\lambda \in \mathbb{N}}$. Let $\tilde{\mathcal{H}}_{\lambda}=\left\{\mathcal{H}: X \longrightarrow\{0,1\}^{\lambda}: X \in \chi_{\lambda}\right\}$ and $\tilde{\mathcal{H}}=\left\{\tilde{\mathcal{H}}_{\lambda}\right\}_{\lambda \in \mathbb{N}}$. Note that $X$ is identified by the description of $\mathcal{H}$. Let $\mathcal{A}_{\text {PreIm }}$ be an adversary playing the following game.

\begin{tabular}{l} 
Pre.Game \\
1) Step 1: $\mathcal{H} \longrightarrow \tilde{\mathcal{H}}_{\lambda}$ \\
2) Step 2: $\boldsymbol{y} \longrightarrow\{0,1\}^{\lambda}$ \\
3) Step 3: $\boldsymbol{x} \longrightarrow \mathcal{A}_{\text {PreIm }}(\mathcal{H}, \boldsymbol{y})$ such that $\boldsymbol{x} \in X$ \\
\hline
\end{tabular}

Fig. 12. Preimage game

$\mathcal{A}_{\text {PreIm }}$ wins the game when $\mathcal{H}(\boldsymbol{x})=\boldsymbol{y}$ and the advantage of $\mathcal{A}_{\text {PreIm }}$ is the probability that it wins Pre.Game for a given $\mathcal{H} \longrightarrow \tilde{\mathcal{H}}_{\lambda}$ and $\boldsymbol{y} \in\{0,1\}^{\lambda}$. We say that $\tilde{\mathcal{H}}$ is Target Preimage free with regard to $\chi$ when the advantage $\epsilon_{\text {PreIm }}$ of $\mathcal{A}_{\text {PreIm }}$ is negligible.

\section{Security of the McEliece encryption with Fujisaki-Okamoto conversion}

For the IND-CCA security of McEliece's scheme described in Figure 1, we need the following definition:

Definition 2: ( $\gamma$-uniformity [16]) A public key encryption scheme $\Pi$ is called $\gamma$-uniform and $\mathcal{R}$ be the set where the randomness to be used in the (probabilistic) encryption is chosen. For a given key-pair $(p k, s k), \boldsymbol{x}$ be a plaintext and a string $\boldsymbol{y}$, we define

$$
\gamma(\boldsymbol{y})=\operatorname{Pr}\left[\boldsymbol{r} \stackrel{\$}{\leftarrow} \mathcal{R}: \boldsymbol{y}=\mathcal{E}_{p k}(\boldsymbol{x}, \boldsymbol{r})\right]
$$


where the notation $\mathcal{E}_{p k}(\boldsymbol{x}, \boldsymbol{r})$ makes the role of the randomness $\boldsymbol{r}$ explicit. We say that $\Pi$ is $\gamma$-uniform if, for any key-pair $(p k, s k)$, any plaintext $\boldsymbol{x}$ and any ciphertext $\boldsymbol{y}, \gamma(\boldsymbol{x}, \boldsymbol{y}) \leq \gamma$ for a certain $\gamma \in \mathbb{R}$.

We now can state the following lemma.

Lemma 1:

The McEliece scheme with the Fujisaki-Okamoto conversion described in Figure 1 is $\gamma$ uniform with

$$
\gamma=\frac{1}{2^{\tilde{k}}\left(\begin{array}{l}
n \\
t
\end{array}\right)}
$$

Proof: For any vector $\boldsymbol{y} \in \mathbb{F}_{2}^{n_{r}}$, either $\boldsymbol{y}$ is a word at distance $t$ from the code $\mathcal{C}$ of generator matrix $\mathbf{G}_{p k, r}$, or it isn't. When $\boldsymbol{y}$ is not a distance $t$ of $\mathcal{C}$, the probability for it to be a valid ciphertext is equal to 0 . Else there is only one choice for $\boldsymbol{r}$ and $\boldsymbol{e}$ such that $\boldsymbol{y}=\boldsymbol{r} \mathbf{G}_{p k, r} \oplus \boldsymbol{e}$, i.e.,

$$
\operatorname{Pr}(d(\boldsymbol{y}, \mathcal{C}))=t)=\frac{1}{2^{\tilde{k}}\left(\begin{array}{c}
n_{r} \\
t
\end{array}\right)}
$$

Theorem 5: Under Assumptions 1 and 3, the McEliece scheme with the Fujisaki-Okamoto conversion described in Figure 1 is IND-CCA2 secure.

Proof: In Figure 1, the symmetric encryption used is the XOR function which is a one-time pad. Under Assumptions 1 and 3, the old McEliece encryption scheme is one-way secure. Therefore according to Theorem 12 of [33], the McEliece scheme with the Fujisaki-Okamoto conversion is IND-CCA2 secure. 